\title{
An Analytical Solution for Acoustic Emission Source Location for Known P Wave Velocity System
}

\author{
Longjun Dong, ${ }^{1}$ Xibing $\mathrm{Li}^{1}{ }^{1}$ and Gongnan $\mathrm{Xie}^{2}$ \\ ${ }^{1}$ School of Resources and Safety Engineering, Central South University, Changsha 410083, China \\ ${ }^{2}$ School of Mechanical Engineering, Northwestern Polytechnical University, Xian 710129, China \\ Correspondence should be addressed to Longjun Dong; rydong001@csu.edu.cn
}

Received 31 December 2013; Revised 24 February 2014; Accepted 25 February 2014; Published 27 March 2014

Academic Editor: Massimo Scalia

Copyright (c) 2014 Longjun Dong et al. This is an open access article distributed under the Creative Commons Attribution License, which permits unrestricted use, distribution, and reproduction in any medium, provided the original work is properly cited.

\begin{abstract}
This paper presents a three-dimensional analytical solution for acoustic emission source location using time difference of arrival (TDOA) measurements from $N$ receivers, $N \geqslant 5$. The nonlinear location equations for TDOA are simplified to linear equations, and the direct analytical solution is obtained by solving the linear equations. There are not calculations of square roots in solution equations. The method solved the problems of the existence and multiplicity of solutions induced by the calculations of square roots in existed close-form methods. Simulations are included to study the algorithms' performance and compare with the existing technique.
\end{abstract}

\section{Introduction}

The solution of the problem of locating a signal source using time difference of arrival (TDOA) measurements has numerous applications in aerospace, surveillance, structural health, nondestructive testing, navigation, industrial process, speaker location, machine condition, the monitoring of nuclear explosions, and mining induced areal seismology [1-17]. Many authors have discussed and faced numerous problems connected with the location of acoustic emission. The time difference of arrival TDOA method, based on estimates of time delay for a correlated signal as detected by spatially distributed sensor elements in an array, remains a commonly used technique for source location [5].

The TDOAs are proportional to the differences in sensorsource range, called range differences (RDs). Conventionally, the source location is estimated from the intersection of a set of hyperboloids defined by the RD measurements and the known sensor locations [18]. The inverse problem for TDOA source location is usually solved by an iterative technique such as nonlinear least squares, minimum error, or an optimization method in recognizing that the equations are nonlinear with respect to source location [19-23]. Kalman filtering has also been used to iteratively solve the TDOA source location problem for microphone speaker location $[24,25]$. Although these iterative algorithms are resilient to varying extents to errors in arrival time data, they may be computationally expensive. This is a key consideration in some real-time applications [21, 22].

Closed-form solutions are usually less computationally burdensome than iterative, nonlinear minimization, or the ML method and achieve good accuracy [18]. Several closedform analytical solutions to the TDOA source location problem have been developed [4, 21, 26, 27]. Closed-form solutions have been found in terms of intersecting spheres of distance from each sensor to an arbitrarily located source spherical intersection method, for a monitoring array of four or more sensors, in some cases admitting dual source location solutions $[28,29]$. A spherical interpolation (SI) method based on linear least-squares equation error minimization with respect to source range has also been developed [30]. The two-dimensional (2D) TDOA source location problem has also been solved [21,31]. A technique has been successfully developed by Kundu et al. [32] for localizing acoustic source in anisotropic plates that avoids the need to solve a system of nonlinear equations. The advantage of the technique is that the knowledge of the wave velocity in isotropic or anisotropic plate is not required in two-dimensional structure, but the 
three-dimensional conditions are not considered. A family of exact solutions for 2D TDOA based on arrival times at four sensors in a plane is also known [33]. A linear intersection (LI) estimator for 3D source location has also been proposed, based on the closest intersection of source bearing lines calculated for four sensor element subarrays under a far-field assumption $[22,34]$. This method is claimed to closely model the behavior of the maximum likelihood estimator and to be consistently less sensitive to noise in the time differences than the SI method. A similar but less heuristic closed-form solution based on the far-field assumption has also been proposed [4]. A simultaneous analytical solution for the spatial coordinates of a source and the redundant radial distance of the source/time of origin of the signal has also been reported in the literature [29]. A direct and short derivation of an algorithm based on the closed-form solution of the nonlinear equations for emitter location using time difference of arrival (TDOA) is given [18], and the influences of imaginary roots on solutions were discussed. Spencer developed closed-form solutions for both two-dimensional and three-dimensional source locations by formulating the TDOA equations in, respectively, polar and spherical coordinate systems, with the radial direction coincident with the assumed geodesic path of signal propagation to a reference sensor [5].

Nevertheless, the existence and multiplicity of the closedform solutions are affected by square root calculation in solution equation. In this paper, we simplify the nonlinear location equations for TDOA to linear equations and develop a unique three-dimensional analytical solution without having square root calculation.

\section{Analytical Solutions}

An acoustic emission source is located at source $(x, y, z)$ and the 5 geosensors are located at $S_{1}\left(x_{1}, y_{1}, z_{1}\right), S_{2}\left(x_{2}, y_{2}, z_{2}\right)$, $S_{3}\left(x_{3}, y_{3}, z_{3}\right), S_{4}\left(x_{4}, y_{4}, z_{4}\right)$, and $S_{5}\left(x_{5}, y_{5}, z_{5}\right)$. The equations governing the position of acoustic emission source and sensors are

$$
\begin{aligned}
& \left(x_{1}-x\right)^{2}+\left(y_{1}-y\right)^{2}+\left(z_{1}-z\right)^{2}=v^{2} t_{0}^{2}, \\
& \left(x_{2}-x\right)^{2}+\left(y_{2}-y\right)^{2}+\left(z_{2}-z\right)^{2}=v^{2}\left(t_{0}+t_{12}\right)^{2}, \\
& \left(x_{3}-x\right)^{2}+\left(y_{3}-y\right)^{2}+\left(z_{3}-z\right)^{2}=v^{2}\left(t_{0}+t_{13}\right)^{2}, \\
& \left(x_{4}-x\right)^{2}+\left(y_{4}-y\right)^{2}+\left(z_{4}-z\right)^{2}=v^{2}\left(t_{0}+t_{14}\right)^{2}, \\
& \left(x_{5}-x\right)^{2}+\left(y_{5}-y\right)^{2}+\left(z_{5}-z\right)^{2}=v^{2}\left(t_{0}+t_{15}\right)^{2},
\end{aligned}
$$

where $t_{0}$ is acoustic wave propagation time from source to the nearest sensor. The signal arrives first to the nearest sensor. After identification of the nearest sensor and calculation of propagation time delay between nearest sensor and other sensors, the sensors are numbered. The nearest sensor from source is numbered as sensor $1\left(S_{1}\right) . t_{12}, t_{13}, t_{14}$, and $t_{15}$ are the propagation time delay between sensor 1 and sensors 2 , 3,4 , and 5, respectively. $v$ is the $\mathrm{P}$ wave velocity. Equations (1)-(5) represent spheres, with the center at respective sensor position, passing through the source. Any two of the spheres
(1)-(5) intersect and the source is located on the intersecting circle. The equation of the intersecting plane for the spheres (1) and (2) can be obtained by taking difference of (1) and (2). This equation of intersecting plane is given in (6). In the following equations, $l_{i}$ is a constant:

$$
2\left(x_{2}-x_{1}\right) x+2\left(y_{2}-y_{1}\right) y+2\left(z_{2}-z_{1}\right) z+2 t_{12} v^{2} t_{0}=l_{1},
$$

where $l_{1}=x_{2}^{2}-x_{1}^{2}+y_{2}^{2}-y_{1}^{2}+z_{2}^{2}-z_{1}^{2}-v^{2} t_{12}^{2}$.

Similarly, the equations for the intersecting planes for spheres (1) and (3), for spheres (1) and (4), and for spheres (1) and (5) are obtained by taking difference of (1) and (3) and (1) and (4), as well as (1) and (5), respectively. These equations are given in (7), (8), and (9), respectively:

$$
\begin{aligned}
& 2\left(x_{3}-x_{1}\right) x+2\left(y_{3}-y_{1}\right) y \\
& \quad+2\left(z_{3}-z_{1}\right) z+2 v^{2} t_{13} t_{0}=l_{2}, \\
& 2\left(x_{4}-x_{1}\right) x+2\left(y_{4}-y_{1}\right) y \\
& \quad+2\left(z_{4}-z_{1}\right) z+2 v^{2} t_{14} t_{0}=l_{3}, \\
& 2\left(x_{5}-x_{1}\right) x+2\left(y_{5}-y_{1}\right) y \\
& \quad+2\left(z_{5}-z_{1}\right) z+2 v^{2} t_{15} t_{0}=l_{4},
\end{aligned}
$$

where $l_{2}=x_{3}^{2}-x_{1}^{2}+y_{3}^{2}-y_{1}^{2}+z_{3}^{2}-z_{1}^{2}-v^{2} t_{13}^{2}, l_{3}=x_{4}^{2}-x_{1}^{2}+y_{4}^{2}-$ $y_{1}^{2}+z_{4}^{2}-z_{1}^{2}-v^{2} t_{14}^{2}$, and $l_{4}=x_{5}^{2}-x_{1}^{2}+y_{5}^{2}-y_{1}^{2}+z_{5}^{2}-z_{1}^{2}-v^{2} t_{15}^{2}$.

Equations (6), (7), (8), and (9) constitute a set of linear equations, which were rewritten as

$$
\begin{aligned}
l_{5} x+l_{6} y+l_{7} z+l_{8} t_{0} & =l_{1}, \\
l_{9} x+l_{10} y+l_{11} z+l_{12} t_{0} & =l_{2}, \\
l_{13} x+l_{14} y+l_{15} z+l_{16} t_{0} & =l_{3}, \\
l_{17} x+l_{18} y+l_{19} z+l_{20} t_{0} & =l_{4},
\end{aligned}
$$

where $l_{5}=2\left(x_{2}-x_{1}\right), l_{6}=2\left(y_{2}-y_{1}\right), l_{7}=2\left(z_{2}-z_{1}\right), l_{8}=$ $2 t_{12} v^{2}, l_{9}=2\left(x_{3}-x_{1}\right), l_{10}=2\left(y_{3}-y_{1}\right), l_{11}=2\left(z_{3}-z_{1}\right)$, $l_{12}=2 t_{13} v^{2}, l_{13}=2\left(x_{4}-x_{1}\right), l_{14}=2\left(y_{4}-y_{1}\right), l_{15}=2\left(z_{4}-z_{1}\right)$, $l_{16}=2 t_{14} v^{2}, l_{17}=2\left(x_{5}-x_{1}\right), l_{18}=2\left(y_{3}-y_{1}\right), l_{19}=2\left(z_{5}-z_{1}\right)$, and $l_{20}=2 t_{15} v^{2}$.

Therefore, the $x, y, z$, which can be obtained by solving the linear equations, can be expressed and simplified as

$$
\begin{gathered}
x=\left[l _ { 1 } \left(l_{10} l_{16} l_{19}-l_{11} l_{16} l_{18}-l_{12} l_{14} l_{19}\right.\right. \\
\left.+l_{12} l_{15} l_{18}-l_{10} l_{15} l_{20}+l_{11} l_{14} l_{20}\right) \\
+l_{6}\left(l_{15} l_{2} l_{20}-l_{16} l_{19} l_{2}+l_{12} l_{19} l_{3}\right. \\
\left.-l_{11} l_{20} l_{3}+l_{11} l_{16} l_{4}-l_{12} l_{15} l_{4}\right) \\
+l_{7}\left(l_{16} l_{18} l_{2}-l_{14} l_{2} l_{20}-l_{12} l_{18} l_{3}\right. \\
\left.\quad-l_{10} l_{20} l_{3}-l_{10} l_{16} l_{4}+l_{12} l_{14} l_{4}\right) \\
+l_{8}\left(l_{14} l_{19} l_{2}-l_{15} l_{18} l_{2}-l_{10} l_{19} l_{13}\right. \\
\left.\left.+l_{11} l_{18} l_{3}+l_{10} l_{15} l_{4}-l_{11} l_{14} l_{4}\right)\right]
\end{gathered}
$$




$$
\begin{aligned}
& \times\left[l _ { 5 } \left(l_{10} l_{16} l_{19}-l_{11} l_{16} l_{18}-l_{12} l_{14} l_{19}\right.\right. \\
& \left.+l_{12} l_{15} l_{18}-l_{10} l_{15} l_{20}+l_{11} l_{14} l_{20}\right) \\
& +l_{6}\left(l_{11} l_{16} l_{17}+l_{12} l_{13} l_{19}-l_{12} l_{15} l_{17}-l_{11} l_{13} l_{20}\right) \\
& +l_{7}\left(l_{10} l_{13} l_{20}-l_{10} l_{16} l_{17}-l_{12} l_{13} l_{18}+l_{12} l_{14} l_{17}\right) \\
& +l_{8}\left(l_{10} l_{15} l_{17}-l_{10} l_{13} l_{19}+l_{11} l_{13} l_{18}-l_{11} l_{14} l_{17}\right) \\
& +l_{9}\left(l_{15} l_{6} l_{20}-l_{19} l_{16} l_{6}+l_{7} l_{16} l_{18}\right. \\
& \left.\left.-l_{20} l_{14} l_{17}+l_{19} l_{14} l_{8}-l_{15} l_{18} l_{8}\right)\right]^{-1}, \\
& y=\left[l _ { 1 } \left(l_{11} l_{16} l_{17}-l_{12} l_{13} l_{19}-l_{12} l_{15} l_{17}\right.\right. \\
& \left.+l_{11} l_{13} l_{20}-l_{16} l_{19} l_{9}+l_{15} l_{19} l_{20}\right) \\
& +l_{5}\left(l_{16} l_{2} l_{19}-l_{5} l_{20} l_{2}+l_{12} l_{19} l_{3}\right. \\
& \left.-l_{11} l_{20} l_{3}+l_{11} l_{16} l_{4}-l_{12} l_{15} l_{4}\right) \\
& +l_{7}\left(l_{16} l_{17} l_{2}-l_{13} l_{2} l_{20}-l_{12} l_{17} l_{3}-l_{12} l_{13} l_{4}\right) \\
& +l_{9}\left(l_{20} l_{3} l_{7}-l_{16} l_{4} l_{7}-l_{19} l_{8} l_{3}-l_{15} l_{14} l_{8}\right) \\
& \left.+l_{8}\left(l_{13} l_{19} l_{2}-l_{15} l_{17} l_{2}-l_{11} l_{17} l_{3}+l_{11} l_{13} l_{4}\right)\right] \\
& \times\left[l _ { 5 } \left(l_{10} l_{16} l_{19}-l_{11} l_{16} l_{18}-l_{12} l_{14} l_{19}\right.\right. \\
& \left.+l_{12} l_{15} l_{18}-l_{10} l_{15} l_{20}+l_{11} l_{14} l_{20}\right) \\
& +l_{6}\left(l_{11} l_{16} l_{17}+l_{12} l_{13} l_{19}-l_{12} l_{15} l_{17}-l_{11} l_{13} l_{20}\right) \\
& +l_{7}\left(l_{10} l_{13} l_{20}-l_{10} l_{16} l_{17}-l_{12} l_{13} l_{18}+l_{12} l_{14} l_{17}\right) \\
& +l_{8}\left(l_{10} l_{15} l_{17}-l_{10} l_{13} l_{19}+l_{11} l_{13} l_{18}-l_{11} l_{14} l_{17}\right) \\
& +l_{9}\left(l_{15} l_{6} l_{20}-l_{19} l_{16} l_{6}+l_{7} l_{16} l_{18}\right. \\
& \left.\left.-l_{20} l_{14} l_{17}+l_{19} l_{14} l_{8}-l_{15} l_{18} l_{8}\right)\right]^{-1}, \\
& z=\left[l _ { 5 } \left(l_{18} l_{16} l_{2}-l_{14} l_{2} l_{20}-l_{12} l_{18} l_{3}\right.\right. \\
& \left.+l_{10} l_{3} l_{20}-l_{16} l_{10} l_{4}+l_{12} l_{14} l_{4}\right) \\
& +l_{6}\left(l_{13} l_{20} l_{2}-l_{16} l_{2} l_{17}+l_{12} l_{17} l_{3}-l_{12} l_{13} l_{4}\right) \\
& +l_{8}\left(l_{14} l_{17} l_{2}-l_{13} l_{2} l_{18}-l_{10} l_{17} l_{3}+l_{10} l_{13} l_{4}\right) \\
& +l_{9}\left(l_{1} l_{14} l_{20}-l_{1} l_{16} l_{18}-l_{20} l_{6} l_{3}\right. \\
& \left.\left.+l_{16} l_{6} l_{4}+l_{18} l_{3} l_{8}-l_{14} l_{8} l_{4}\right)\right] \\
& \times\left[l _ { 5 } \left(l_{10} l_{16} l_{19}-l_{11} l_{16} l_{18}-l_{12} l_{14} l_{19}\right.\right. \\
& \left.+l_{12} l_{15} l_{18}-l_{10} l_{15} l_{20}+l_{11} l_{14} l_{20}\right) \\
& +l_{6}\left(l_{11} l_{16} l_{17}+l_{12} l_{13} l_{19}-l_{12} l_{15} l_{17}-l_{11} l_{13} l_{20}\right) \\
& +l_{7}\left(l_{10} l_{13} l_{20}-l_{10} l_{16} l_{17}-l_{12} l_{13} l_{18}+l_{12} l_{14} l_{17}\right) \\
& +l_{8}\left(l_{10} l_{15} l_{17}-l_{10} l_{13} l_{19}+l_{11} l_{13} l_{18}-l_{11} l_{14} l_{17}\right)
\end{aligned}
$$

$$
\begin{aligned}
& +l_{9}\left(l_{15} l_{6} l_{20}-l_{19} l_{16} l_{6}+l_{7} l_{16} l_{18}\right. \\
& \left.\left.\quad-l_{20} l_{14} l_{17}+l_{19} l_{14} l_{8}-l_{15} l_{18} l_{8}\right)\right]^{-1} .
\end{aligned}
$$

Equation (10) can also rewrite as

$$
\mathbf{A S}=\mathbf{B},
$$

where

$$
\mathbf{A}=\left[\begin{array}{cccc}
l_{5} & l_{6} & l_{7} & l_{8} \\
l_{9} & l_{10} & l_{11} & l_{12} \\
l_{13} & l_{14} & l_{15} & l_{16} \\
l_{17} & l_{18} & l_{19} & l_{20}
\end{array}\right], \quad \mathbf{S}=\left[\begin{array}{c}
x \\
y \\
z \\
t_{0}
\end{array}\right], \quad \mathbf{B}=\left[\begin{array}{l}
l_{1} \\
l_{2} \\
l_{3} \\
l_{4}
\end{array}\right] .
$$

The $x, y$, and $z$ can be also obtained by solving the linear systems. The proposed method is suitable for known wave velocity system. The closed-form solution for unknown wave velocity system is reported in [35].

\section{Validated Examples}

3.1. Example 1: Simulations for Sound Sources. For example, consider five receivers and a speed of sound of $330 \mathrm{~m} / \mathrm{s}$. The Cartesian coordinates of five sensors are S1 (250.25, 450.17, and 150.28), S2 (290.11, 180.13, and 140.18), S3 (150.35, 190.21, and 30.33), S4 (380.38, 250.28, and 98.15), and S5 $(500.55,350.25$, and 210.45$) \mathrm{m}$. The two source locations with Cartesian coordinates of P1 $(310.75,200.33$, and 125.11) and P2 $(210.88,290.99$, and 175.89) $\mathrm{m}$ and the original times of two sources are $0 \mathrm{~s}$. The trigger times are obtained according to the distance formula between spatial two points as well as distance and velocity formula. The trigger times of five receivers (S1-S5) of P1 are 0.782697, 0.098713, 0.565408, 0.272225 , and $0.777218 \mathrm{~s}$, respectively. The trigger times of five receivers (S1-S5) of P2 are 0.502922, 0.426859, 0.566984, 0.578392 , and $0.902068 \mathrm{~s}$, respectively.

To validate the proposed method, the coordinates of receivers and their trigger time were used to calculate $l_{i}(i=$ $1,2, \ldots, 20)$ according to $(7)-(10)$. Then, the calculated $l_{i}(i=$ $1,2, \ldots, 20)$ were substituted in (11) to resolve the source location. The calculated results of sources $\mathrm{P} 1$ and P2 are $(310.7499,200.3302$, and 125.1101$)$ and $(210.8797,290.9899$, and 175.8904) $\mathrm{m}$, respectively. Results show that the calculated coordinates of P1 and P2 are consistent with authentic results.

3.2. Example 2: Simulations of a Mine Acoustic Emission Location System and Location Error Analysis. A numerical test for an acoustic emission/microseismic monitoring system of a mine was used to validate the proposed method and analyze location errors. The locations of five sensors are shown in Figure 1, and the coordinates are $(200,500$, and $300)$, (300, 100, and 100), (400, 350, and 280), (600, 250, and 250), and (700, 200, and 180). The locations of acoustic emission/microseismic sources E1, E2, E3, E4, and E5 are 1 (250, 300, and 120), 2 (310, 450, and 130), 3 (450, 260, and 110), 

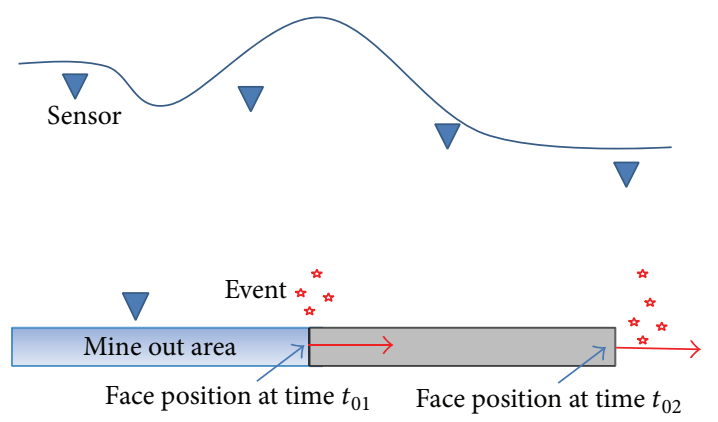

(a) Locations of sensors and sources

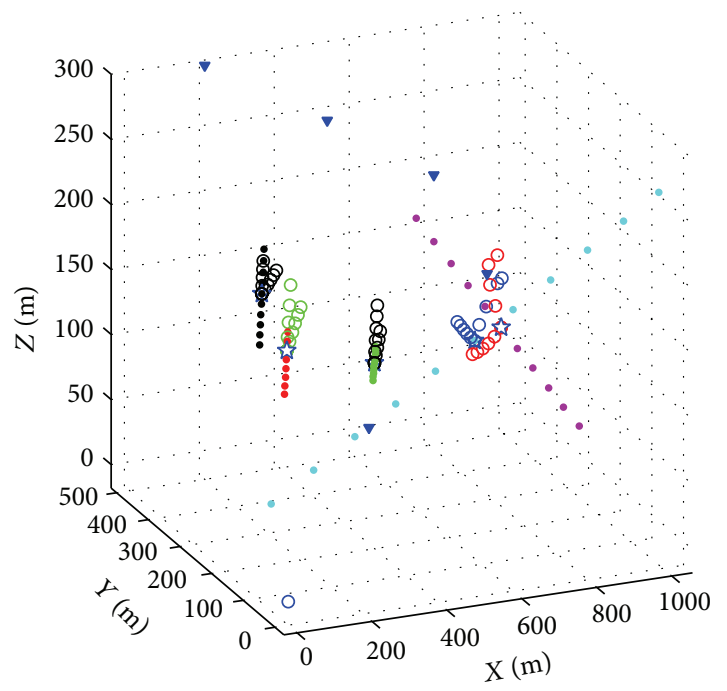

$\begin{array}{ll}\rightarrow \text { Sensor } & \circ \text { E5-N } \\ \text { is Event } & \cdot \text { E1-A } \\ \circ \text { E1-N } & \cdot \text { E2-A } \\ \circ \text { E2-N } & \cdot \text { E3-A } \\ \circ \text { E3-N } & \cdot \text { E4-A } \\ \circ \text { E4-N } & \cdot \text { E5-A }\end{array}$

(b) Spatial locations of sensors and sources

FIGURE 1: Sketch map of acoustic emission/microseismic source location: $\mathrm{A}$ indicates analytical solution; $\mathrm{N}$ indicates numerical solution.

$4(650,180$, and 135$)$, and $5(780,250$, and 125$)$, respectively, and the unit is m; P-wave velocity is $5000 \mathrm{~m} / \mathrm{s}$. Errors of wave velocity and arrivals were discussed.

Firstly, the acoustic emission sources are located by proposed method using true wave velocity. The results are shown in Figure 2. It can be seen that the calculated results are consistent with the true locations. Therefore, it can be concluded that proposed method is reasonable. To investigate the location errors induced by errors of velocities, the method STT was used to compare and analyze. The locations of acoustic emissions by STT method without considering the errors of velocities and arrivals are consistent with true locations (Figure 2). The errors of absolute distant are shown in Figure 2. It also can be seen that the results of STT are consistent with true locations and results of the proposed analytical solution method. It is proved that the STT method

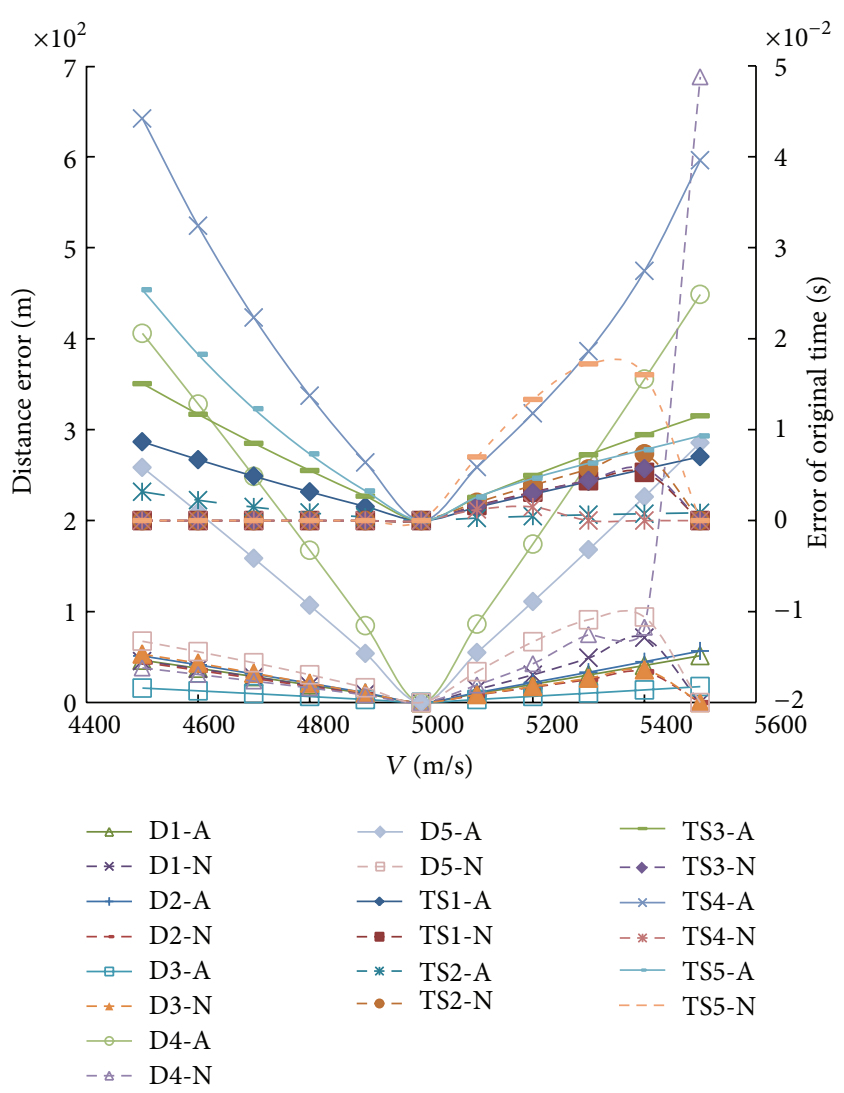

FIGURE 2: Errors of distance error from located source to real source and original time induced by insufficient know velocity error: D indicates absolute distant error; TS indicates original time error.

is stable and reliable. The intervals of velocity errors are from $4500 \mathrm{~m} / \mathrm{s}$ to $5500 \mathrm{~m} / \mathrm{s}$. True velocity is $5000 \mathrm{~m} / \mathrm{s}$. The variable interval is $200 \mathrm{~m} / \mathrm{s}$. Location results with analytical solutions and numerical methods STT are shown in Figure 1. The absolute distance errors between the real coordinates and calculated coordinates are shown in Figure 2 under different levels of velocity error. The left vertical axis is the absolute distance error. The original time errors are also plotted in the right vertical axis of Figure 2. It can be seen from positioning results of Figures 1 and 2 that the positioning accuracy is affected by the velocity value. In view of the spatial distribution of positions, symmetrical changes of velocity will result in a positioning results symmetry spatial location using analytical solution positioning, since analytical solutions are based on the results of each positioning operation parameters of the true value. The location errors are completely dependent on the operator error parameters which are offset from the true value. Symmetrical changes of velocity will induce irregular spatial position using numerical iterative method, STT. If velocity error is large, it may cause serious positioning errors, which will locate a serious deviation from the true value, such as the E4-N. It can be seen from Figure 2 that higher positioning analytical location method requires measurement data with high quality, but when measurement data is reasonable, it can obtain accurate positioning results. Throughout all of located microseismic sources, it can be 


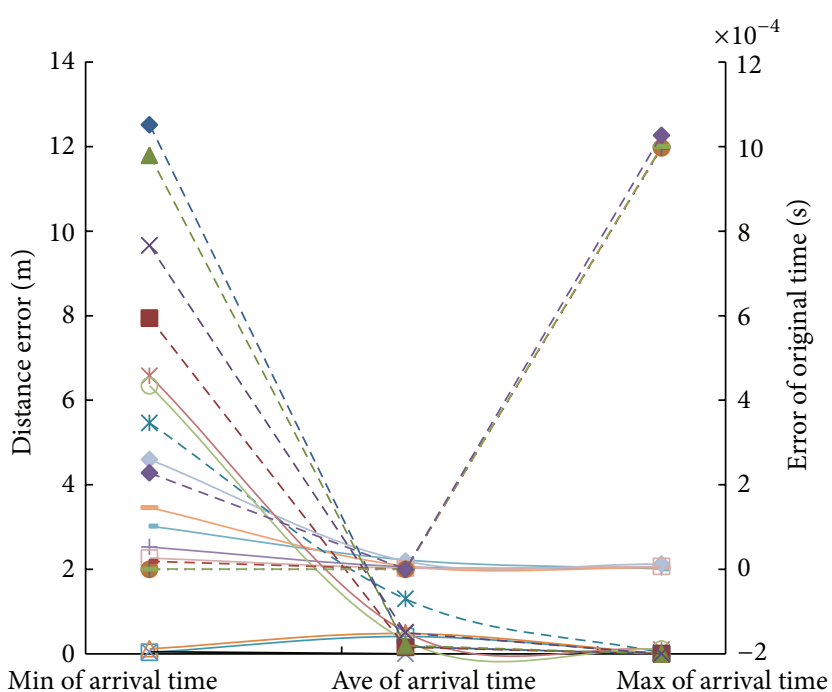

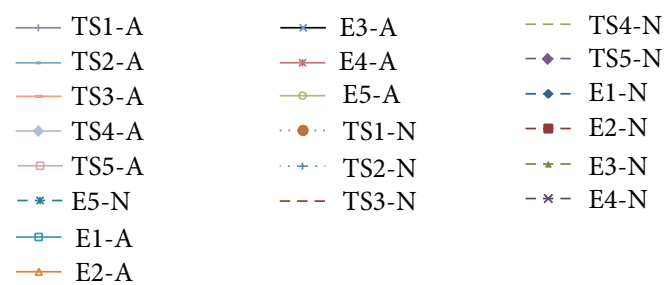

FIGURE 3: Errors of Distance and original time induced by arrival time reading error: the left $y$ axil expressing the distance error from located sources to real sources and right $y$ axil expressing the error of original time, E indicates absolute distant error; TS indicates original time errors; A indicates analytical solutions; and N indicates STT results.

easily found that the greatest absolute distance error is from the numerical solution of STT. A velocity error of $500 \mathrm{~m} / \mathrm{s}$ can result in a big absolute distance error of $700 \mathrm{~m}$ using STT, while the positioning error of the analytical solution is $450 \mathrm{~m}$.

Secondly, to investigate the location errors induced by errors of arrivals, the method STT was also used to compare and analyze. The error of arrivals is limited between $-1000 \mu \mathrm{s}$ and $+1000 \mu \mathrm{s}$. The 1000 errors are generated by the random function of Microsoft Excel; then arrivals with errors were obtained by true arrivals plus generated errors. In this study, the minimum, average, and maximum of arrivals with errors were used to investigate the location errors induced by arrival errors. The calculated results are shown in Figure 3. The absolute distance error is expressed in the left vertical axis. Original time error is expressed in the right vertical axis. It can be clearly seen in Figure 3 that arrival errors also significantly affect the location errors. The absolute distant errors and original errors of STT are always larger than those of analytical method. The maximums of absolute distant errors of STT and analytical method are $12.5 \mathrm{~m}$ and $6.5 \mathrm{~m}$, respectively. The maximums of original time errors of STT and analytical method are $0.001 \mathrm{~s}$ and $0.00023 \mathrm{~s}$, respectively.

\section{Conclusions}

We simplify the nonlinear location equations for TDOA to linear equations. Based on solving simplified linear equations, we obtain a unique three-dimensional analytical solution for acoustic emission source location using TDOA measurements from $N$ receivers, $N \geqslant 5$. There are no calculations of square roots in the solution equations of the proposed method. The problems of the existence and multiplicity induced by calculations of square roots in existed close-form methods were solved successfully. Simulations are included to study the algorithms' performance and compare with existing STT method.

\section{Conflict of Interests}

The authors declare that there is no conflict of interests regarding the publication of this paper.

\section{Acknowledgments}

The authors gratefully acknowledge the financial support of National Natural Science Foundation of China (41272304, 51209236), National Basic Research (973) Program of China (2010CB732004), China Scholarship Council (CSC), and Australian center for Geomechanics.

\section{References}

[1] K. L. Rens, T. J. Wipf, and F. W. Klaiber, "Review of nondestructive evaluation techniques of civil infrastructure," Journal of Performance of Constructed Facilities, vol. 11, no. 4, pp. 152160, 1997.

[2] S. J. Spencer, "The two-dimensional source location problem for time differences of arrival at minimal element monitoring arrays," Journal of the Acoustical Society of America, vol. 121, no. 6, pp. 3579-3594, 2007.

[3] Q. Y. Li, L. J. Dong, X. B. Li, Z. Q. Yin, and X. L. Liu, "Effects of sonic speed on location accuracy of acoustic emission source in rocks," Transactions of Nonferrous Metals Society of China, vol. 21, pp. 2719-2726, 2011.

[4] H. Liu and E. Milios, "Acoustic positioning using multiple microphone arrays," Journal of the Acoustical Society of America, vol. 117, no. 5, pp. 2772-2782, 2005.

[5] S. J. Spencer, "Closed-form analytical solutions of the time difference of arrival source location problem for minimal element monitoring arrays," Journal of the Acoustical Society of America, vol. 127, no. 5, pp. 2943-2954, 2010.

[6] D. G. Aggelis, "Classification of cracking mode in concrete by acoustic emission parameters," Mechanics Research Communications, vol. 38, no. 3, pp. 153-157, 2011.

[7] D. G. Aggelis, T. Shiotani, S. Momoki, and A. Hirama, "Acoustic emission and ultrasound for damage characterization of concrete elements," ACI Materials Journal, vol. 106, no. 6, pp. 509514, 2009.

[8] D. G. Aggelis and T. Shiotani, "Experimental study of surface wave propagation in strongly heterogeneous media," Journal of the Acoustical Society of America, vol. 122, no. 5, pp. EL151EL157, 2007.

[9] D. G. Aggelis, T. Shiotani, T. P. Philippidis, and D. Polyzos, "Stress wave scattering: friend or enemy of non destructive 
testing of concrete?" Journal of Solid Mechanics and Materials Engineering, vol. 2, pp. 397-408, 2008.

[10] D. G. Aggelis, T. Shiotani, and M. Terazawa, "Assessment of construction joint effect in full-scale concrete beams by acoustic emission activity," Journal of Engineering Mechanics, vol. 136, no. 7, pp. 906-912, 2009.

[11] T. Shiotani, D. G. Aggelis, and O. Makishima, "Global monitoring of large concrete structures using acoustic emission and ultrasonic techniques: case study,' Journal of Bridge Engineering, vol. 14, no. 3, pp. 188-192, 2009.

[12] L.-J. Dong, X.-B. Li, and K. Peng, "Prediction of rockburst classification using Random Forest," Transactions of Nonferrous Metals Society of China, vol. 23, no. 2, pp. 472-477, 2013.

[13] L. Dong, X. Li, and L. Tang, "Main influencing factors of microseismic source location accuracy," Science and Technology Review, vol. 31, pp. 26-32, 2013.

[14] L. Dong and X. Li, "A microseismic/acoustic emission source location method using arrival times of PS waves for unknown velocity system," International Journal of Distributed Sensor Networks, vol. 2013, Article ID 307489, 8 pages, 2013.

[15] L. Dong and X. Li, “Three-dimensional analytical solution of acoustic emission or microseismic source location under cube monitoring network," Transactions of Nonferrous Metals Society of China, vol. 22, pp. 3087-3094, 2012.

[16] L. Dong, X. Li, L. Tang, and F. Gong, "Mathematical functions and parameters for microseismic source location without premeasuring speed," Chinese Journal of Rock Mechanics and Engineering, vol. 30, no. 10, pp. 2057-2067, 2011.

[17] L. Dong, X. Li, and G. Xie, "Nonlinear methodologies for identifying seismic event and nuclear explosion using Random Forest, support vector machine, and naive bayes classification," Abstract and Applied Analysis, vol. 2014, Article ID 459137, 8 pages, 2014.

[18] G. Mellen II, M. Pachter, and J. Raquet, "Closed-form solution for determining emitter location using time difference of arrival measurements," IEEE Transactions on Aerospace and Electronic Systems, vol. 39, no. 3, pp. 1056-1058, 2003.

[19] R. Belchamber and M. Collins, "Acoustic emission source location using simplex optimization," Journal of Acoustic Emission, vol. 9, pp. 271-276, 1990.

[20] E. Landis, C. Ouyang, and S. P. Shah, "Acoustic emission source locations in concrete," in Proceedings of the Engineering Mechanics Specialty Conference (ASCE '91), pp. 1051-1055, May 1991.

[21] Y. Chan and K. Ho, "An efficient closed-form localization solution from time difference of arrival measurements," in Proceedings of the IEEE International Conference on Acoustics, Speech, and Signal Processing (ICASSP '94), 1994.

[22] M. S. Brandstein, J. E. Adcock, and H. F. Silverman, "A closed-form location estimator for use with room environment microphone arrays," IEEE Transactions on Speech and Audio Processing, vol. 5, no. 1, pp. 45-50, 1997.

[23] J. H. DiBiase, H. F. Silverman, and M. S. Brandstein, "Robust localization in reverberant rooms," in Microphone Arrays, pp. 157-180, Springer, Berlin, Germany, 2001.

[24] S. Gannot and T. G. Dvorkind, "Microphone array speaker localizers using spatial-temporal information," Eurasip Journal on Applied Signal Processing, vol. 2006, Article ID 59625, 17 pages, 2006.

[25] U. Klee, T. Gehrig, and J. McDonough, "Kalman filters for time delay of arrival-based source localization," Eurasip Journal on
Applied Signal Processing, vol. 2006, Article ID 12378, 15 pages, 2006.

[26] R. O. Schmidt, "A new approach to geometry of range difference location," IEEE Transactions on Aerospace and Electronic Systems, vol. 8, no. 6, pp. 821-835, 1972.

[27] S. J. Arrowsmith, M. D. Arrowsmith, M. A. H. Hedlin, and B. Stump, "Discrimination of delay-fired mine blasts in Wyoming using an automatic time-frequency discriminant," Bulletin of the Seismological Society of America, vol. 96, no. 6, pp. 2368-2382, 2006.

[28] H. C. Schau and A. Z. Robinson, "Passive source localization employing intersecting spherical surfaces from time-of-arrival differences," IEEE Transactions on Acoustics, Speech, and Signal Processing, vol. 35, no. 8, pp. 1223-1225, 1987.

[29] J. L. Spiesberger, "Hyperbolic location errors due to insufficient numbers of receivers," The Journal of the Acoustical Society of America, vol. 109, no. 6, pp. 3076-3079, 2001.

[30] J. O. Smith and J. S. Abel, "Closed-form least-squares source location estimation from range-difference measurements," IEEE Transactions on Acoustics, Speech, and Signal Processing, vol. 35, no. 12, pp. 1661-1669, 1987.

[31] X. B. Li and L. J. Dong, "Comparison of two methods in acoustic emission source location using four sensors without measuring sonic speed," Sensor Letters, vol. 9, no. 5, pp. 2025-2029, 2011.

[32] T. Kundu, H. Nakatani, and N. Takeda, "Acoustic source localization in anisotropic plates," Ultrasonics, vol. 25, pp. 740746, 2012.

[33] R. Duraiswami, D. Zotkin, and L. Davis, "Exact solutions for the problem of source location from measured time differences of arrival," The Journal of the Acoustical Society of America, vol. 106, p. 2277, 1999.

[34] M. S. Brandstein, J. E. Adcock, and H. F. Silverman, "Closedform method for finding source locations from microphonearray time-delay estimates," in Proceedings of the 20th International Conference on Acoustics, Speech, and Signal Processing, pp. 3019-3022, May 1995.

[35] X. Li and L. Dong, "An efficient closed-form solution for acoustic emission source location in three-dimensional structures," AIP Advances, vol. 4, no. 2, p. 027110, 2014. 


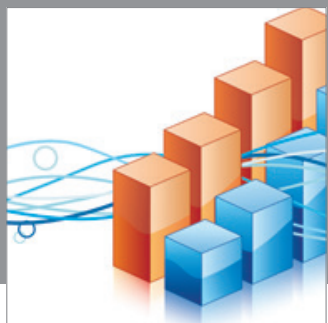

Advances in

Operations Research

mansans

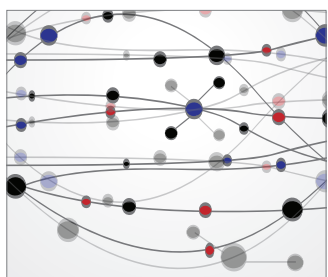

The Scientific World Journal
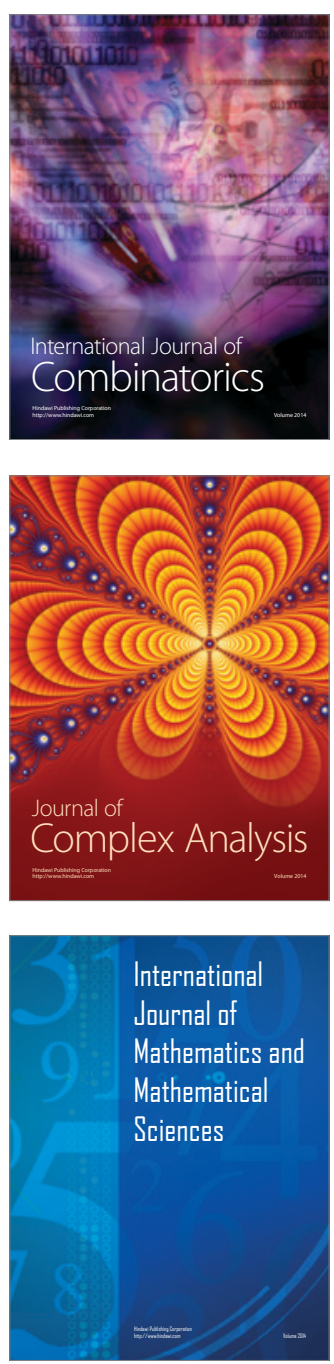
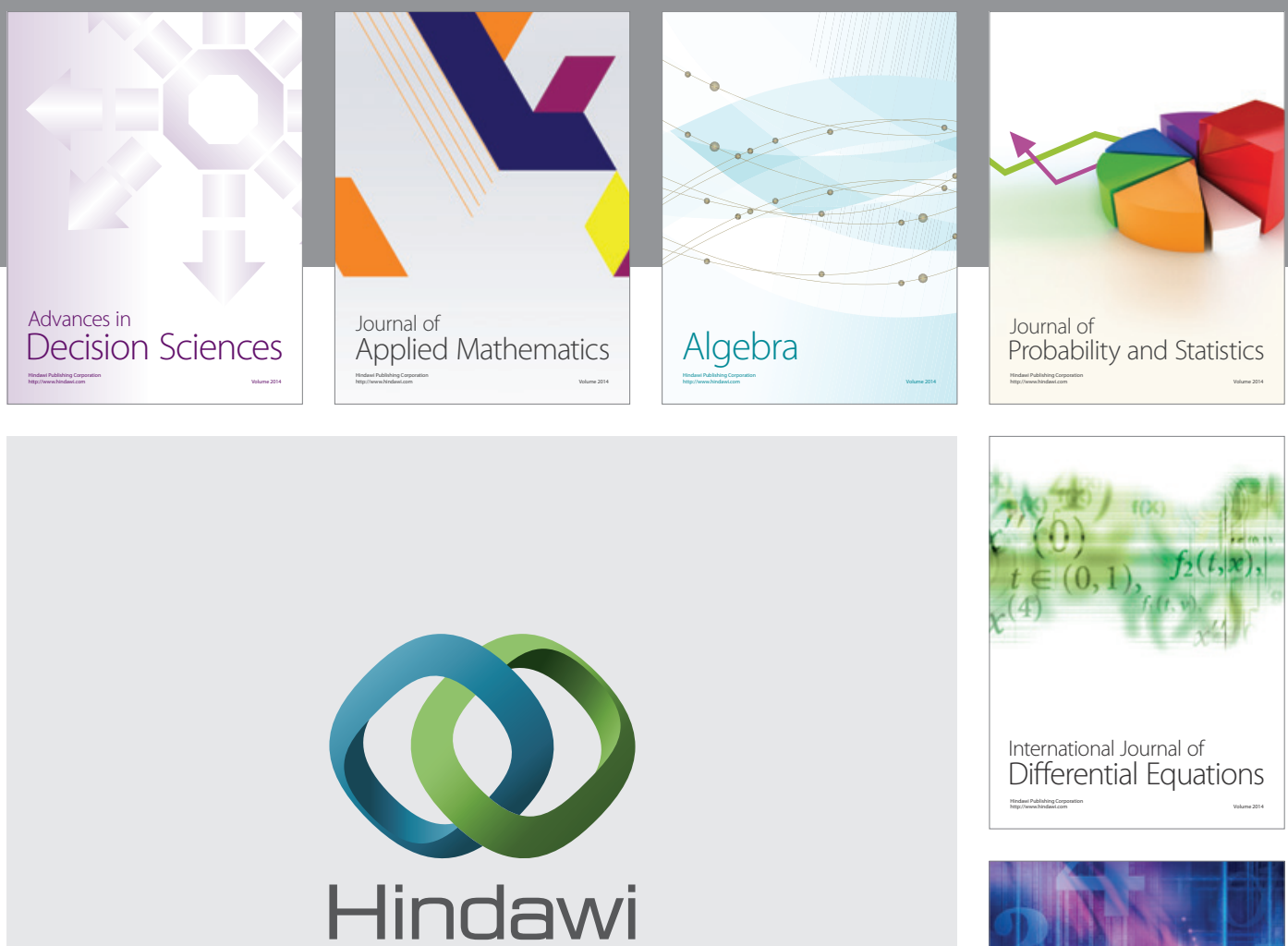

Submit your manuscripts at http://www.hindawi.com
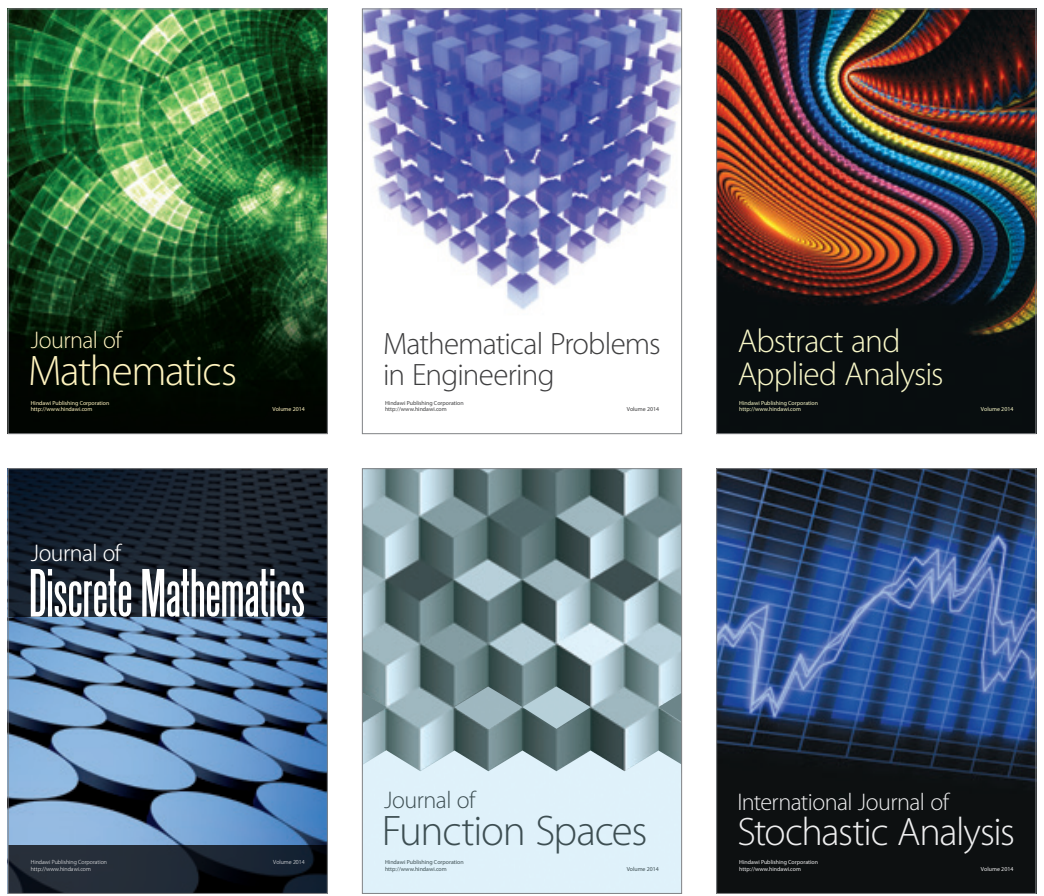

Journal of

Function Spaces

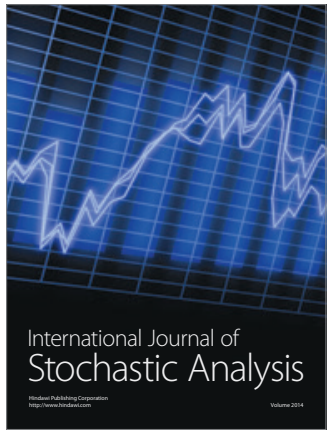

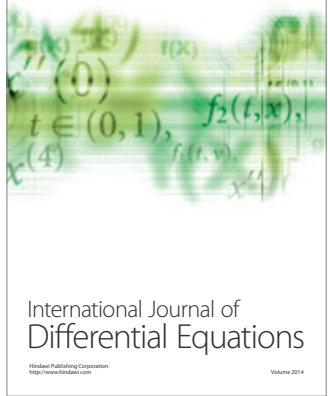
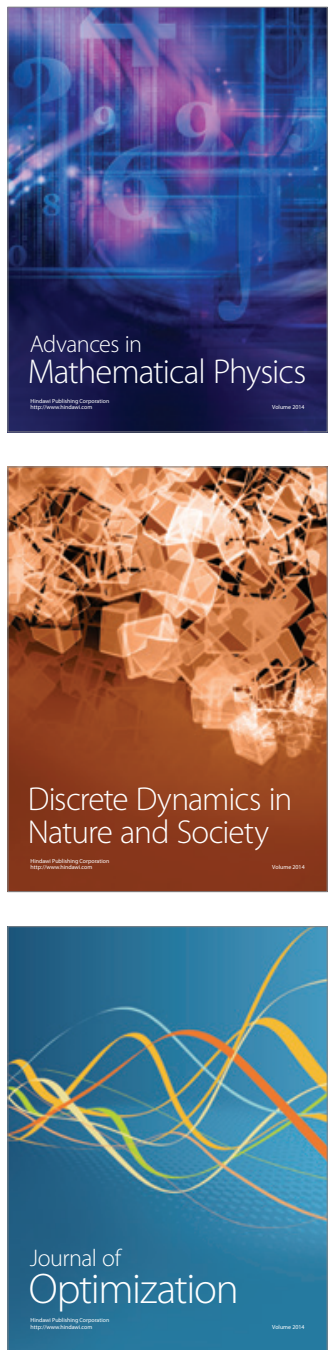